\title{
Preoperative intravenous meloxicam for moderate-to-severe pain in the immediate post-operative period: a Phase IIIb randomized clinical trial in 55 patients undergoing primary open or laparoscopic colorectal surgery with bowel resection and/or anastomosis
}

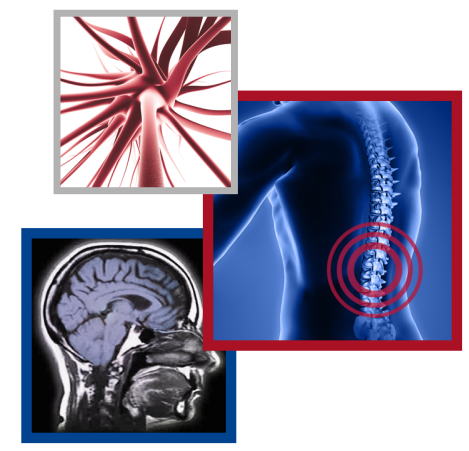
\author{
Libby K Black ${ }^{4}$ \\ ${ }^{1}$ Department of Surgery, Tulane University, New Orleans, LA, 70112, USA \\ ${ }^{2}$ Department of Surgery, Tampa General Hospital, Tampa, FL, 33606, USA \\ ${ }^{3}$ IQVIA, Falls Church, VA, 22042, USA \\ ${ }^{4}$ Baudax Bio Inc., Malvern, PA, 19355, USA \\ ${ }^{5}$ Clinical Statistics Consulting, Blue Bell, PA, 19422, USA \\ *Author for correspondence: Tel.: +1 484395 2440; rmack@baudaxbio.com \\ ${ }^{\ddagger}$ At the time, this work was conducted
}

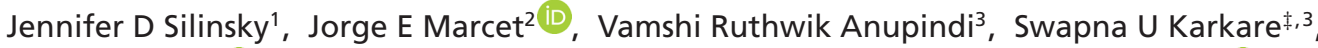
Drishti R Shah ${ }^{3}$ iD, Randall J Mack*,4, Stewart W McCallum4, Wei Du , Alex Freyer4 iD \&

\section{Practice points}

- This randomized, double-blind, multicenter, placebo-controlled study evaluated the safety and efficacy of meloxicam IV as part of an enhanced recovery after surgery (ERAS) protocol in 55 subjects undergoing colorectal surgery.

- Meloxicam IV was generally well tolerated; rates of adverse events and events of special interest were 85 versus $93 \%$ and 30 versus $21 \%$, respectively, in the meloxicam IV and placebo groups. The most common treatment-emergent adverse events in both study groups were nausea and vomiting.

- Meloxicam IV was associated with $35 \%$ lower opioid consumption versus placebo (from end of surgery to discharge, $29.2 \pm 5.2 \mathrm{mg}$ vs $45.2 \pm 5.2 \mathrm{mg}$, respectively; $\mathrm{p}=0.03$ ).

- Meloxicam IV was associated with significant differences versus placebo for pain intensity as measured using the Brief Pain Inventory on postoperative day 1 ( $4.0 \pm 2.0$ vs $5.3 \pm 1.9$ for placebo; $p=0.03$ ), length of stay (time from end of surgery to discharge, $77.0 \pm 7.7$ vs $104.6 \pm 9.0 \mathrm{~h} ; \mathrm{p}=0.03$ ) and functional end points (i.e., reduced times to first bowel sound [ $8.9 \pm 2.1$ vs $21.7 \pm 4.0 \mathrm{~h} ; \mathrm{p}=0.02$ ], first flatus [37.7 \pm 2.8 vs $50.1 \pm 4.5 \mathrm{~h} ; \mathrm{p}=0.03$ ] and first bowel movement [51.5 \pm 5.3 vs $62.4 \pm 5.1 \mathrm{~h} ; \mathrm{p}=0.02]$ ).

Aim: Evaluate safety/efficacy of intravenous meloxicam in a colorectal enhanced recovery after surgery protocol. Methods: Adults undergoing primary open or laparoscopic colorectal surgery with bowel resection and/or anastomosis received meloxicam IV $30 \mathrm{mg}(\mathrm{n}=27)$ or placebo $(\mathrm{n}=28)$ once daily beginning 30 min before surgery. Results: Adverse events: meloxicam IV, 85\%; placebo, 93\%. Adverse events commonly associated with opioids: 41 versus $61 \%$ - including nausea (33 vs 50\%), vomiting (19 vs $18 \%$ ) and ileus (4 vs 18\%). Wound healing satisfaction scores (physician-rated), clinical laboratory findings and vital signs were similar in both groups. No anastomotic leaks were reported. Opioid consumption, postoperative pain intensity, length of stay and times to first bowel sound, first flatus and first bowel movement were significantly lower with meloxicam IV versus placebo. Most subjects $(>92 \%)$ were satisfied with postoperative pain medication. Conclusion: Meloxicam IV was generally well tolerated and associated with decreased opioid consumption, lower resource utilization and functional benefits.

Clinical Trial Registration: NCT03323385 (ClinicalTrials.gov) 
First draft submitted: 6 August 2020; Accepted for publication: 23 September 2020; Published online: 23 October 2020

Keywords: acute pain $\bullet$ colorectal surgery $\bullet$ healthcare resource utilization $\bullet$ intravenous meloxicam $\bullet$ safety $\bullet$ tolerability

Enhanced recovery after surgery (ERAS) protocols are multidisciplinary pathways designed to optimize patient care during the perioperative period and to accelerate postoperative recovery [1-3]. ERAS pathways include interventions throughout the preoperative, perioperative and postoperative periods. Benefits of these protocols include improved pain control, reduced length of stay (LOS), reduced costs, decreased complications and increased patient satisfaction $[1,2,4]$. ERAS protocols have been used for many years [5,6], and there is now a significant supporting evidence base for their effectiveness [7] such that they have become standard of care [8].

Pain control is an important component of ERAS protocols during colorectal surgery [9,10], with effective pain control facilitating recovery and mobilization and minimizing surgery-related complications [10]. Although opioids are effective for pain, they are associated with a number of adverse events (AEs) and complications, such as constipation, respiratory depression, pruritus and dependence $[9,11,12]$.

Nonsteroidal anti-inflammatory drugs (NSAIDs) have well-demonstrated safety and efficacy for perioperative pain and are associated with improved patient satisfaction, decreased opioid requirements and decreased AEs commonly associated with opioid use [11,13,14]. Meloxicam is an NSAID that has a prolonged half-life $(20-24 \mathrm{~h})$ and, at a $15-\mathrm{mg}$ oral dose, is associated with a preferential inhibition of COX-2, resulting in a decreased risk of gastrointestinal AEs compared with nonselective NSAIDs [15]. A formulation of intravenous meloxicam (meloxicam IV; Anjeso ${ }^{T M}$ ) that utilizes a novel nanocrystal formulation has been approved by the US FDA for the management of moderate-to-severe pain alone or in combination with other analgesics, with the recommended dose being $30 \mathrm{mg}$ once daily [16]. The recommended dose was based on several dose-ranging Phase II trials and confirmed in Phase III trials that assessed the safety and efficacy of meloxicam IV administered following hard-tissue [17-19] or soft-tissue surgery [19-21]. Because of delayed onset of analgesia, meloxicam IV alone is not recommended for use when rapid onset of analgesia is required [16].

The objective of this study was to evaluate the safety and efficacy of perioperative administration of meloxicam IV, compared with placebo, in subjects undergoing colorectal surgery. The effect of meloxicam IV on postoperative recovery (including opioid use) and healthcare resource utilization (including incidence of readmission, hospital LOS, total costs of hospital stay) was also reported.

\section{Materials \& methods}

Study design

This randomized, double-blind, multicenter, placebo-controlled study was conducted at ten clinical sites in adult subjects undergoing open or laparoscopic colorectal surgery. Subjects were assigned randomly (1:1) to receive meloxicam IV $30 \mathrm{mg}$ or placebo. Randomization was performed via a computer-generated randomization scheme that produced a block randomization stratified by center and planned incision type (open vs laparoscopic). Subjects, investigators and staff members were blinded to block size and the assigned study treatment. Subjects were expected to stay at the study site for $48-72 \mathrm{~h}$ or as long as inpatient care was considered clinically appropriate. The initial study cohort was to include approximately 50 subjects, with the potential for additional cohorts following review of data from cohort 1 . The study was designed and monitored in accordance with the ethical principles of Good Clinical Practice and in accordance with the Declaration of Helsinki. The protocol was approved by the institutional review boards of participating institutions, and all subjects provided written informed consent.

\section{Subjects}

Eligible for inclusion were males and females aged 18 through 80 years who were planning to undergo a primary (no repeat procedures) open or laparoscopic colorectal surgery, with bowel resection and/or anastomosis and who were expected to require IV analgesia, remain in an inpatient setting for at least $48-72 \mathrm{~h}$ and receive at least two doses of study medication. Subjects also were required to be nonpregnant, use effective contraception, and have a body mass index $\leq 40 \mathrm{~kg} / \mathrm{m}^{2}$ with a performance status that allowed for carrying out normal activities of daily life without limitations. Excluded from participation were subjects whose planned surgical procedure included resection below the peritoneal reflection, was related to acute diverticulitis, or was associated with an emergency procedure. In addition, chronic opioid users were excluded as their pain is difficult to treat and they would have a 
different utilization of opioids than non-chronic users. Other exclusion criteria were an allergy/hypersensitivity to eggs or any NSAID, a planned procedure expected to last $>10 \mathrm{~h}$, a myocardial infarction within 12 months, any significant renal, hepatic, cardiovascular, metabolic, neurologic, psychiatric, respiratory condition or any clinically significant abnormal laboratory values. Moreover, subjects were not allowed to have gastrointestinal ulceration or bleeding within 6 months; subjects could not have a current known bleeding disorder, or a clinically significant 12-lead electrocardiogram (ECG) abnormality. Subjects could not have received chronic opioid therapy (i.e., daily use of opioids for $\geq 30$ days) or $>5$ days of opioid use within 30 days prior to screening. Disallowed medications included NSAIDs (within $48 \mathrm{~h}$ ), herbal medications/supplements associated with increased bleeding risk (ginkgo biloba, garlic, ginger, ginseng, hawthorn, fish oil, dong quai, feverfew and vitamin E) within 7 days; subjects could not be receiving treatment with lithium, or furosemide plus either angiotensin converting enzyme inhibitor or angiotensin receptor blocker. A complete list of inclusion and exclusion criteria is provided in Supplementary data.

\section{Study drug administration}

Subjects received either meloxicam IV $30 \mathrm{mg}$ or placebo, given as an IV bolus over approximately $15 \mathrm{~s}$, with the first dose administered approximately $30 \mathrm{~min}$ before the start of surgery. Additional doses were administered every $24 \pm 1 \mathrm{~h}$ from the first dose until discharge or until IV analgesia was no longer clinically appropriate; this stopping point was designed to reflect clinical practice. Surgeries were completed using an appropriate anesthesia and analgesic regimen, according to the clinical practice of the surgeon and within the guidelines of the study's ERAS protocol. Venous thromboembolism prophylaxis was administered before and after surgery, according to standard practice. Adjunctive measures specified in the ERAS protocol for the pre-, peri- and postoperative periods are summarized in Table 1. Choice of postoperative rescue analgesia (intravenous morphine or patient-controlled morphine) was left to the discretion of the study site. Conversion to oral analgesia (oxycodone) was made once patients were tolerating liquid intake, with morphine IV bolus administered if needed for supplemental analgesia. Patient discharge was not dictated by the study protocol but left to the investigator's judgment. Upon discharge, patients were provided an analgesic care regimen for pain management that was standard of care for that institution.

\section{Assessments/end points}

Safety

The primary objective was to assess the safety and tolerability of meloxicam IV. AEs were assessed throughout treatment and at post-discharge follow-up visits, and all AEs were assessed by the investigator for intensity and relationship to study medication. Serious adverse events (SAEs), AEs of special interest (e.g., hepatobiliary, renal, cardiovascular, thrombotic, bleeding, wound healing and injection-site events), and AEs commonly associated with opioid use (including, but not limited to, somnolence, respiratory depression, hypoventilation, hypoxia, dry mouth, nausea/vomiting, constipation, sedation, confusion, pruritus, urinary retention and postoperative ileus) were also identified. Investigator satisfaction with surgical wound healing was rated on an 11-point scale, from 0 (completely unsatisfied) to 10 (completely satisfied). Safety assessments also included physical examination and obtaining vital signs, and clinical laboratory parameters (as assessed by the investigator), all of which were done at screening, prior to surgery and at the end of treatment (i.e., day of last study dose +1 day). Blood samples were collected from a subset of subjects at check-in prior to surgery on day 1 , and once daily thereafter until last study day +1 , for analysis of inflammatory markers including: CRP, TNF- $\alpha$, IL-1B, IL-4, IL-6, IL-8, IL-10 and IL-18. Collection and analysis of the blood samples for levels of inflammatory markers was considered an exploratory analysis, as the postoperative profile of these markers has not been well established and the clinical implications of changes in these levels is not fully understood.

\section{Opioid consumption \& efficacy end points}

All opioid consumption (IV morphine or oral oxycodone $5 \mathrm{mg}$ ) was recorded and converted to the IV morphine equivalent dose (IVMED, mg; for oxycodone $0.5 \times$ dose [mg]) [22]. Opioid use in the postoperative period was evaluated over several time intervals, including h 0 (i.e., end of surgery, defined as the time of last suture) to discharge, h $0-24$, h $0-48$, h 24-48, h 0-72 and h 48-72. Twenty-four-hour intervals were evaluated because the duration of the meloxicam IV dosing interval is $24 \mathrm{~h}$. Other end points included time to first rescue medication; various pain outcomes, including the Patient Global Assessment of pain control (PGA) and the brief pain inventory (BPI); and satisfaction with pain management. The PGA was assessed on a 5-point scale, ranging from poor to excellent. The BPI assessed several dimensions of pain and function, with the two primary domains being pain intensity and 


\section{Table 1. Enhanced recovery after surgery protocol.}

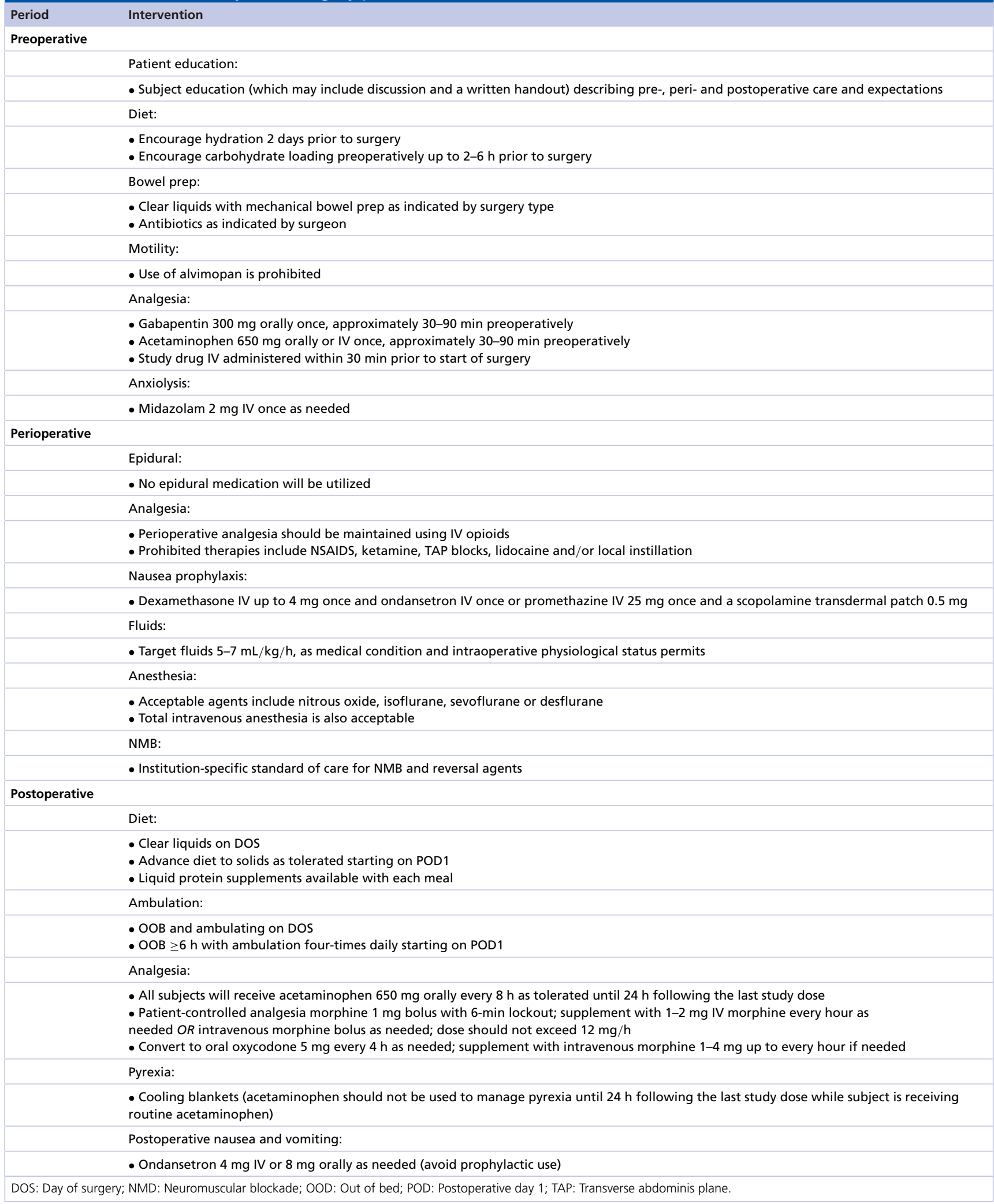




\begin{tabular}{|c|c|}
\hline \multirow[t]{7}{*}{ Period } & Intervention \\
\hline & Fluids: \\
\hline & - Fluids to be managed per subjects' individual needs \\
\hline & Motility: \\
\hline & $\begin{array}{l}\text { - Gum chewing is prohibited } \\
\text { - Use of alvimopan is prohibited }\end{array}$ \\
\hline & Tubes/drains: \\
\hline & $\begin{array}{l}\text { - Orogastric/nasogastric tubes should be removed in the operating room or on DOS } \\
\text { - Urinary catheters should be removed on or before POD1 }\end{array}$ \\
\hline
\end{tabular}

pain interference. The BPI was conducted at screening; both PGA and BPI were attained $24 \mathrm{~h}$ following the end of surgery, then every $24 \mathrm{~h}$ through $24 \mathrm{~h}$ after the last dose of study drug. Subject satisfaction with postoperative pain management was assessed on a seven-point Likert scale, ranging from completely dissatisfied to completely satisfied.

\section{Healthcare resource utilization \& functional end points}

Various healthcare resource utilization and functional end points were assessed, including the incidence of readmission, hospital LOS (expressed as h and days to discharge), total costs of hospital stay (not including cost of study drug), time to first ambulation, pain intensity on first assisted ambulation and time to first bowel sound, first flatus and first bowel movement.

\section{Statistical methods}

An initial sample size of 50 subjects was selected empirically without a formal power calculation. This sample size was selected in order to perform an interim analysis to assess the effect size, from which the final sample size would be determined. Following review of an interim analysis of safety and efficacy for this cohort, the Data Review Committee recommended not to enroll a second cohort as the meloxicam IV group demonstrated a statistically significant reduction in terms of LOS and opioid use compared with the placebo group.

The intent-to-treat (ITT) population included all randomized subjects. The modified intent-to-treat (mITT) population included all subjects who received at least one injection of study drug and underwent their scheduled surgery. The mITT population was used to assess all efficacy variables for each study group. Safety end points were summarized descriptively without formal inferential statistics. Any differences between the groups for opioid consumption and efficacy end points were evaluated using analysis of covariance models, and Cox proportional hazards analysis was used to evaluate treatment effect in time-to-event parameters (i.e., time to first opioid rescue mediation use, time to first ambulation, time to return of normal bowel function, time to hospital discharge). Analysis of covariance models were used because the study was a multi-center study in which each site enrolled a small number of patients, all of whom were pooled for analysis. The Cochran-Mantel-Haenszel analysis, controlling for investigational sites, was used to assess between group differences for categorical parameters. Differences between the groups were evaluated via a two-sided, two-sample $t$-test at the 0.05 significance level.

Total costs of hospital stay were captured from UB-04 claim forms and included all charges incurred from admission until the discharge date. A cost:charge ratio of 0.477 was used to convert charges to costs. A generalized linear regression model with log-link and gamma distribution were used to assess the impact of group assignment, total opioid consumption, occurrence of AEs commonly associated with opioid use and pain control (PGA) on hospital LOS and total costs of hospital stay. The results included in these analyses were based on interim analysis of the data. As such, the analyses did not include corrections for multiple comparisons.

The safety population was used for all safety and tolerability assessments and included all subjects who received study medication. Safety end points were analyzed descriptively, without formal statistical tests.

\section{Results}

\section{Subject \& surgical characteristics}

Fifty-seven subjects (27 meloxicam IV, 30 placebo) were eligible and randomized, 55 of whom received $\geq 1$ dose of study drug (Figure 1). Of these, 53 subjects completed the trial; two subjects (one in each arm) decided 


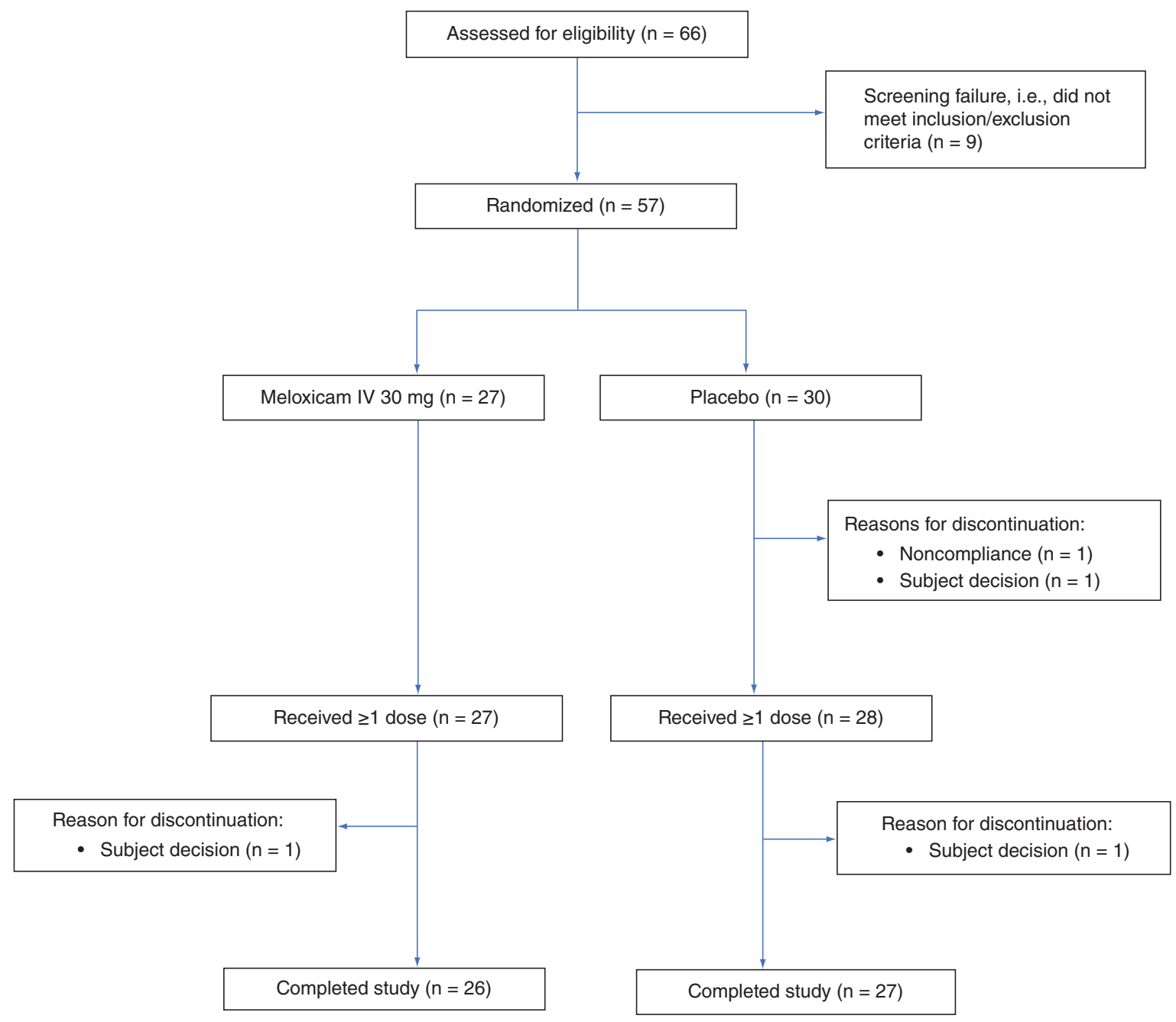

Figure 1. Consolidated Standards of Reporting Trials patient flow diagram.

to discontinue. Baseline demographics and clinical/surgical characteristics are listed in Table 2. Demographic characteristics were generally similar between groups but there tended to be more surgeries for cancer or polyps in the meloxicam IV group and more surgeries for diverticular disease in the placebo group. Surgery types mostly consisted of bowel resection and colectomies, with a few varied secondary procedures included. Most subjects in the meloxicam IV and placebo groups received two (37 and 29\%, respectively) or three (56 and 61\%, respectively) doses of study medication. There were no meaningful differences in protocol deviations or in the timing of study drug administration between groups. A total of one (4\%) and ten $(37 \%)$ patients in the meloxicam IV group and four (14\%) and $16(57 \%)$ patients in the placebo group had used the proton-pump inhibitors omeprazole and pantoprazole, respectively, prior to or during the study.

Safety

The majority of the study population (89\%) experienced $\geq 1$ treatment-emergent AE (TEAE). The proportion of subjects who experienced a TEAE was 85 and $93 \%$, respectively, in the meloxicam IV and placebo groups and the overall number of TEAEs per subject was 2.4 for meloxicam IV and 3.4 for placebo. The most common TEAEs in both study groups were nausea and vomiting (Table 3), and the majority of these were mild or moderate in intensity. SAEs were reported for three subjects (11\%) in the meloxicam IV group and four subjects (14\%) in the placebo group. SAEs in the meloxicam IV group included ileus, incision-site cellulitis and wound dehiscence $(\mathrm{n}=1$ each); SAEs in the placebo group included ileus $(\mathrm{n}=2)$, and hypoglycemia and paranasal sinus benign neoplasm $(\mathrm{n}=1$ each). None of the SAEs was considered related to study medication, and all resolved. 
Table 2. Subject demographics, baseline characteristics and surgical characteristics.

\begin{tabular}{|c|c|c|c|}
\hline Characteristic & Meloxicam IV $30 \mathrm{mg}(\mathrm{n}=27)$ & Placebo $(n=28)$ & Overall $(n=55)$ \\
\hline Mean age (years) & $58.8(11.2)$ & $60.6(11.1)$ & $59.7(11.1)$ \\
\hline$-\geq 65$ years, $\mathrm{n}(\%)$ & $9(33.3)$ & $12(42.9)$ & $21(38.2)$ \\
\hline - Female, n (\%) & $12(44.4)$ & $11(39.3)$ & $23(41.8)$ \\
\hline \multicolumn{4}{|l|}{ Race, n (\%) } \\
\hline - White & $22(81.5)$ & $23(82.1)$ & $45(81.8)$ \\
\hline - Black or African-American & $4(14.8)$ & $5(17.9)$ & $9(16.4)$ \\
\hline Mean baseline $\mathrm{BMI}\left(\mathrm{kg} / \mathrm{m}^{2}\right)$ & $28.9(5.7)$ & $27.5(4.7)$ & $28.2(5.2)$ \\
\hline \multicolumn{4}{|l|}{ Primary indication for surgery, $\mathrm{n}(\%)$} \\
\hline - Colon cancer & $13(48.1)$ & $9(32.1)$ & $22(40.0)$ \\
\hline - Diverticular disease & $4(14.8)$ & $9(32.1)$ & $13(23.6)$ \\
\hline - Intestinal polyps & $5(18.5)$ & 1 (3.6) & $6(10.9)$ \\
\hline - Other & $3(11.1)$ & $7(25.0)$ & $10(18.2)$ \\
\hline \multicolumn{4}{|l|}{ Actual incision type, $\mathrm{n}(\%)$} \\
\hline - Open & $3(11.1)$ & $2(7.1)$ & $5(9.1)$ \\
\hline - Laparoscopic & $20(74.1)$ & $23(82.1)$ & $43(78.2)$ \\
\hline - Laparoscopic converted to open & $4(14.8)$ & $3(10.7)$ & $7(12.7)$ \\
\hline $\begin{array}{l}\text { Mean time from first dose to surgery } \\
\text { start, } \min (\mathrm{SD})\end{array}$ & $37.4(17.4)$ & $31.2(13.9)$ & $34.3(15.9)$ \\
\hline Mean surgery duration, h(SD) & $2.6(1.3)$ & $2.4(0.96)$ & $2.5(1.1)$ \\
\hline Mean time in PACU, $\min (\mathrm{SD})$ & $98.0(67.0)$ & $110.0(56.0)$ & $104.1(61.4)$ \\
\hline
\end{tabular}

Table 3. Adverse events occurring in $\geq 2$ subjects in either study group.

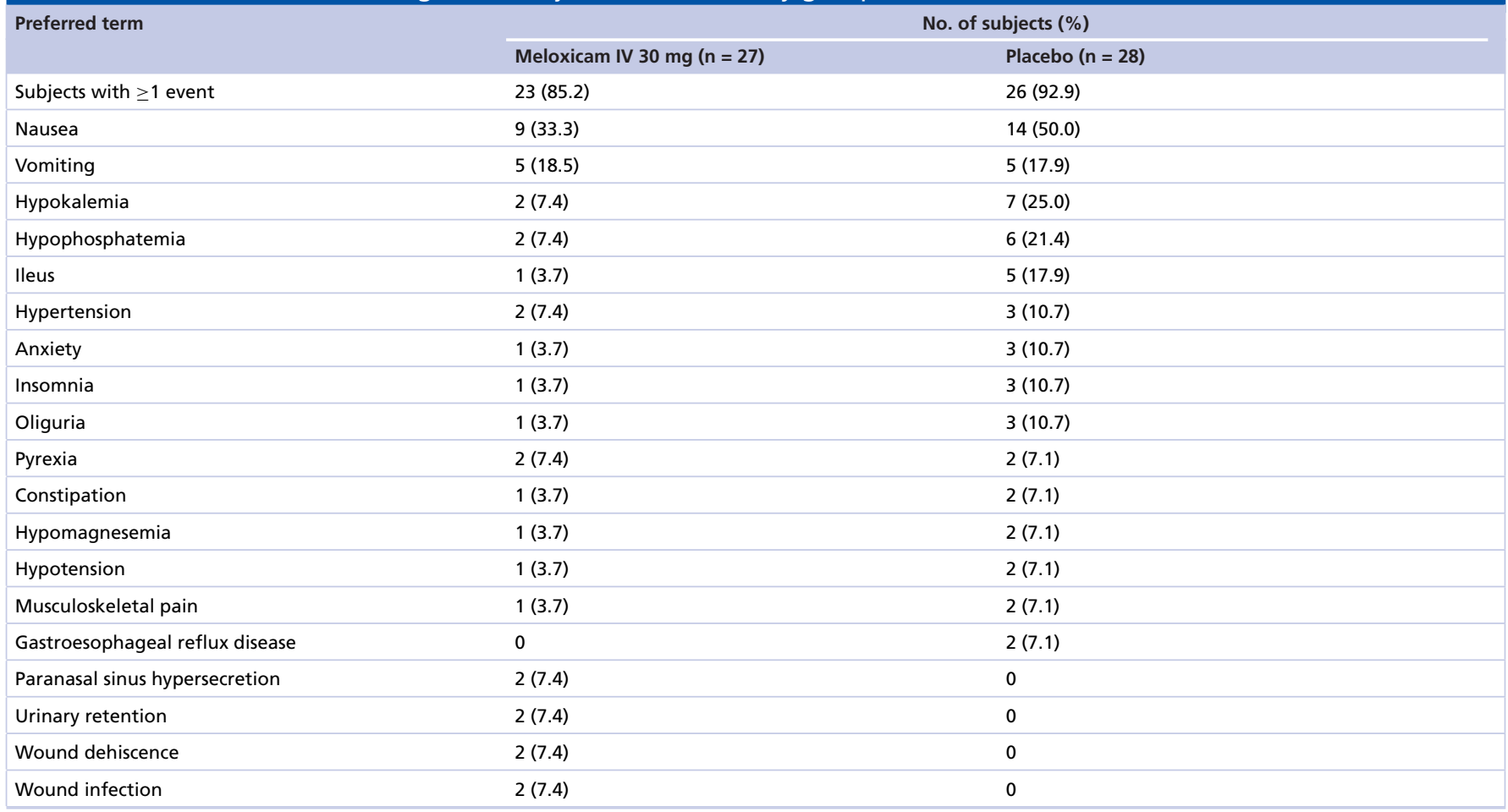


Table 4. Adverse events of special interest occurring in $\geq 1$ subject in either study group.

\begin{tabular}{|c|c|c|}
\hline \multirow{2}{*}{ AEOSI category/preferred term (MedDRA 20.1) } & \multicolumn{2}{|c|}{ No. of subjects (\%) } \\
\hline & Meloxicam IV $30 \mathrm{mg}(\mathrm{n}=27)$ & Placebo $(n=28)$ \\
\hline Subjects with $\geq 1$ AEOSI & 8 (29.6) & $6(21.4)$ \\
\hline \multicolumn{3}{|l|}{ Bleeding } \\
\hline - Incision-site hematoma & 0 & $1(3.6)$ \\
\hline - Post-procedural hemorrhage & $1(3.7)$ & 0 \\
\hline \multicolumn{3}{|l|}{ Cardiovascular } \\
\hline \multicolumn{3}{|l|}{ Hepatic } \\
\hline - Abnormal liver function test & $1(3.7)$ & $1(3.6)$ \\
\hline \multicolumn{3}{|l|}{ Injection-site reactions } \\
\hline - Injection-site pain & $1(3.7)$ & 0 \\
\hline \multicolumn{3}{|l|}{ Renal } \\
\hline - Incision-site swelling & 0 & $1(3.6)$ \\
\hline - Wound dehiscence & $2(7.4)$ & 0 \\
\hline - Wound infection & $2(7.4)$ & 0 \\
\hline
\end{tabular}

Incidence rates of AEs of special interest are shown in Table 4. Eleven meloxicam-treated subjects (41\%) and 17 placebo-treated subjects (61\%) experienced an AE commonly associated with opioid use; the most frequently occurring of these in the meloxicam IV and placebo groups were nausea (33 vs 50\%), vomiting (19 vs 18\%) and ileus ( 4 vs 18\%), respectively. Two subjects experienced wound dehiscence; one event was serious, neither was considered related to study treatment. The SAE of wound dehiscence occurred in a 55-year-old white male with a history of hypertension, colon cancer and postoperative nausea/vomiting. The patient was randomized to meloxicam IV and received his first dose of study drug prior to undergoing open, sigmoid colectomy, and received his last dose 2 days later. Immediately following surgery, the patient experienced postoperative vomiting that resulted in a medically significant wound dehiscence with evisceration of the omentum and a loop of small bowel. The subject underwent surgical closure of abdominal wall dehiscence/evisceration the day following the initial surgery. The investigator considered the event of wound dehiscence to be severe in intensity, not related to study drug, and resolved following the second procedure. The other subject experienced superficial wound dehiscence on day 15 of the study that was considered unrelated to study treatment. The event was mild in severity and resolved.

There were no significant differences between the groups in physician-rated mean surgical wound healing satisfaction scores at hospital discharge and at the follow-up visit. No anastomotic leaks were reported following surgery in any subject. There were no differences between the groups in clinical laboratory findings (hematology, chemistry, coagulation parameters) or vital signs. No clear trends were apparent in the incidence of patients with elevated inflammatory marker levels following surgery.

\section{Opioid consumption \& efficacy end points Opioid consumption}

Opioid consumption (IVMED) was lower in the meloxicam IV group at all time intervals, and the differences versus placebo were statistically significant at some time points (Table 5). Total opioid consumption from $\mathrm{h} 0$ through discharge was $35 \%$ lower in the meloxicam IV group $(29.2 \pm 5.2 \mathrm{mg}$ vs $45.2 \pm 5.2 \mathrm{mg}$ in the placebo group; $\mathrm{p}=$ 0.03 ). The largest difference in opioid consumption occurred on post-surgery day 2 (h 24-48), when meloxicam IV was associated with $49 \%$ lower opioid consumption $(\mathrm{p}=0.01)$. Overall, subjects treated with meloxicam IV received $16.9 \mathrm{mg}$ IVMED fewer opioids than did placebo-treated subjects $(\mathrm{p}=0.04)$. The time from end of surgery to first opioid rescue medication did not differ significantly between the meloxicam IV and placebo groups $(2.0 \pm 0.99$ vs $2.2 \pm 1.1 \mathrm{~h}$, respectively; $\mathrm{p}=0.66)$. 
Table 5. Summary of total opioid consumption (in mg of IV morphine equivalent dose) by time interval.

\begin{tabular}{|lllll|}
\hline $\begin{array}{l}\text { Total opioid consumption (least } \\
\text { square mean [standard error]) } \\
\text { by time interval }\end{array}$ & Meloxicam IV30 $\mathbf{m g}(\mathbf{n}=\mathbf{2 7})$ & Placebo( $\mathbf{n}=\mathbf{2 8})$ & Group mean difference $(\%)$ & -value \\
\hline Hour 0-discharge & $29.2(5.2)$ & $45.2(5.2)$ & $35.3 \%$ & 0.0339 \\
\hline Hour 0-24 & $17.5(5.7)$ & $19.1(2.5)$ & $8.4 \%$ & 0.6500 \\
\hline Hour 24-48 & $6.7(1.7)$ & $13.1(1.7)$ & $49.1 \%$ & 0.0106 \\
\hline Hour 48-72 & $4.3(1.5)$ & $8.3(1.3)$ & $48.0 \%$ & 0.0540 \\
\hline Hour 0-48 & $24.1(3.8)$ & $32.1(3.8)$ & $25.0 \%$ & 0.1393 \\
\hline Hour 0-72 & $27.5(4.5)$ & $39.9(4.5)$ & $31.0 \%$ & 0.0567 \\
\hline Hour 0-EOT & $27.2(4.5)$ & $38.6(4.4)$ & $29.6 \%$ & 0.0730 \\
\hline Overall (total in study) & $49.3(5.7)$ & $66.1(5.7)$ & $25.5 \%$ & 0.0403 \\
\hline
\end{tabular}

EOT: End of treatment; IVMED: IV morphine equivalent dose.

\section{Table 6. Economic and healthcare resource data.}

\begin{tabular}{|c|c|c|c|}
\hline & Meloxicam IV 30 mg $(n=27)$ & Placebo $(n=27)^{\dagger}$ & p-value \\
\hline Time from EOS to discharge, $h$ (SD) & $77.0(7.7)$ & $104.6(9.0)$ & 0.0301 \\
\hline Total hospital LOS, $\mathrm{h}^{\ddagger}$ (SD) & $86.2(42.4)$ & $111.7(48.5)$ & 0.0162 \\
\hline Total hospital costs ${ }^{\S}(\mathrm{SD})$ & $\$ 23,115(\$ 19,013)$ & $\$ 22,682(\$ 14,828)$ & 0.3370 \\
\hline
\end{tabular}

$\dagger$ Missing data from one subject.

$\ddagger$ Reported as number of $\mathrm{h}$ from hospital admission to discharge.

$\S$ A cost-to-charge ratio of 0.477 was applied to convert charges to cost.

EOS: End of surgery; LOS: Length of stay; SD: Standard deviation.

\section{Pain outcomes}

The pain intensity domain of the BPI was significantly lower for meloxicam IV on postoperative day 1 (4.0 \pm 2.0 vs $5.3 \pm 1.9$ for placebo; $\mathrm{p}=0.03$ ). For other BPI time intervals, there were no meaningful differences between the study groups. Most subjects in both the meloxicam IV (92.3\%) and placebo (92.6\%) groups were satisfied with their postoperative pain medication. There were no significant differences in PGA of pain control between the groups.

\section{Healthcare resource utilization \& functional outcomes}

Mean time from end of surgery to actual hospital discharge and mean total LOS were significantly shorter (28 h) in the meloxicam IV group (Table 6 \& Supplementary Table 1). There was no significant difference in total cost of hospital stay between the study groups, although costs in the meloxicam IV group but not the placebo group were driven by outliers. Regression analysis (Supplementary Table 1) demonstrated that increased opioid consumption was associated with increased LOS; every 1-mg increase in opioid consumption from h 0 to discharge was associated with a $0.58-\mathrm{h}$ increase in LOS $(\mathrm{p}=0.0005)$. The presence of an AE commonly associated with opioid use ( $\geq 1$ vs 0 ) was associated with 24.4 more LOS h $(p=0.03)$. Events commonly associated with opioid use were associated with a $16 \%$ increase in total costs, but the increase was not statistically significant $(p=0.32)$. Surgery type (bowel resection vs other) was significantly associated with total costs and LOS, with the highest costs linked to bowel resection.

The mean times to first bowel sound $(8.9 \pm 2.1$ vs $21.7 \pm 4.0 \mathrm{~h} ; \mathrm{p}=0.02)$, first flatus $(37.7 \pm 2.8$ vs $50.1 \pm 4.5 \mathrm{~h} ; \mathrm{p}=0.03)$ and first bowel movement $(51.5 \pm 5.3$ vs $62.4 \pm 5.1 \mathrm{~h} ; \mathrm{p}=0.02)$ were significantly shorter in the meloxicam IV group. Mean times to first ambulation were similar with meloxicam IV and placebo $(13.3 \pm 2.0$ vs $14.0 \pm 1.8 \mathrm{~h}$, respectively; $\mathrm{p}=0.40)$.

\section{Discussion}

Adequate pain relief is an important component of ERAS protocols and is associated with various beneficial effects, including shorter LOS, enhanced recovery, faster mobilization and better patient satisfaction [1-4,23]. Opioids have been a mainstay of perioperative pain control. However, an important goal of ERAS protocols is to reduce opioid consumption, which in turn may lead to decreased known opioid-related risks (e.g., dependence, 
abuse and diversion) and AEs (e.g., nausea/vomiting, impaired gastric motility, constipation and respiratory depression) [24-26].

The current study included patients who were planning to undergo a primary (no repeat procedures) open or laparoscopic colorectal surgery, with bowel resection and/or anastomosis. Patients with resections beyond the peritoneal reflection (e.g., rectal resections and anastomoses) were considered higher risk for anastomotic leaks and were thus excluded. Patients were stratified by incision type rather than by indication for surgery, which resulted in differences in indication between treatment groups; i.e., there were more surgeries for cancer or polyps in the meloxicam IV group and more surgeries for diverticular disease in the placebo group. Although there were insufficient cases to perform a subgroup analysis by incision type, surgery type does not appear likely to affect the primary end point of total opioid consumption. There were insufficient open and open-plus-converted cases to perform an informative subgroup analysis by incision type.

The results demonstrated that, on average, subjects treated with meloxicam IV consumed approximately $16 \mathrm{mg}$ (35\%) fewer opioids compared with placebo subjects from the end of surgery to discharge (in IVMED), and $17 \mathrm{mg}$ fewer opioids overall. The results are consistent with those of meloxicam IV studies in a variety of other surgical populations $[19,21,27]$ and with findings for other NSAIDs in colorectal surgery [14]. For example, a pooled analysis of data from Phase III trials of meloxicam IV for treatment of moderate-to-severe postoperative pain demonstrated that subjects who received meloxicam IV had significantly less opioid consumption in h 0-24, 0-48 and 0-72 following surgery [27]. The reduction in opioid use was largely driven by reductions in opioid use on the second day postoperatively. The reasons for this phenomenon are unknown but may be related to more consistent pain levels and more liberal use of opioids on the first day and/or the anti-inflammatory effect of meloxicam IV. We do not have specific data to confirm any of the aforementioned suppositions.

Assessment of pain outcomes in the present study showed a significant association between meloxicam IV and lower scores for the pain intensity domain of the BPI on postoperative day 1, but not for other parameters that assessed dimensions of pain and functioning. It is noteworthy that this improvement in pain control occurred even though opioid usage in the meloxicam IV group was lower than in the placebo group. These results corroborate those of other meloxicam IV studies in which this treatment was associated with better pain intensity and SPID scores (e.g., SPID $_{24}$ ) than placebo, along with less need for opioid rescue medication (e.g., morphine milligram equivalents [MMEs]) $[17,18,21]$.

NSAIDs have well-known analgesic and anti-inflammatory properties that make them attractive for use in surgical analgesic regimens $[11,14]$. In the current study, meloxicam IV was well tolerated, although $\geq 85 \%$ of patients in both treatment groups had at least one reported TEAE. This relatively high rate of TEAEs is a result of differences in $\mathrm{AE}$ collection in a clinical trial setting compared with the real-world setting (e.g., solicitation vs patient self-report). Overall rates of TEAEs were numerically lower for meloxicam IV, but rates of AEs of special interest were numerically higher in meloxicam-treated patients. The difference in AEs of special interest was largely due to instances of wound dehiscence and wound infection $(n=2$ each) in the meloxicam IV group but not the placebo group. Due to the small study size, these results may not be demonstrated in a real-world setting; although meta-analyses suggest that nonselective, but not selective NSAIDs are associated with an increased risk of anastomotic dehiscence after colorectal surgery [28,29]. We also note that meloxicam IV carries a black box warning for serious risk of cardiovascular and gastrointestinal events.

The decreased effect of meloxicam IV on gastric motility was further evidenced by the significantly shorter times (versus placebo) to first bowel sound, first flatus and first bowel movement. Faster return of normal bowel function is particularly important for patients who undergo colorectal surgery because ileus can occur in approximately $10 \%$ of these patients [9,14]. In addition, slow recovery of bowel function is most often the reason patients require a longer stay in the hospital $[9,14]$. The findings from this study are consistent with those of studies showing that use of NSAIDs helps improve recovery of bowel function after colorectal surgery [14]. Additionally, there was no significant difference between study groups in TEAEs of special interest, including bleeding, renal, cardiovascular, hepatic and wound healing events, and there were no reports of anastomotic leaks for any subject.

The economic analysis showed that meloxicam IV was associated with shorter LOS versus placebo, measured by the number of $\mathrm{h}$ from hospital admission to discharge. Sensitivity analyses demonstrated that shorter LOS for meloxicam IV may be related to lower opioid use and fewer AEs commonly associated with opioid use, as subjects who experienced $\geq 1 \mathrm{AE}$ commonly associated with opioid use had significantly longer hospital stays than those with no events. Total costs of hospital stay were numerically lower for meloxicam IV; however, the differences between study groups were not statistically significant. The trial was not sufficiently powered to detect such differences. 
The study also showed that the presence of an AE commonly associated with opioid use was associated with a significantly longer LOS and numerically higher total costs.

The primary limitation of this study is the small sample size. An interim analysis was planned after 50 completed patients; upon review of the positive results, the decision was made to stop the study early, thus preventing the enrollment being sufficient to demonstrate statistical differences on additional study end points. Also, the number of subjects in each study arm was not identical. This is a reflection of the efforts made to keep the study blinded. Block randomization was used to minimize the probability that the site could discern treatment assignment; block size (four) was not revealed to the site. Randomization was also stratified by incision type and site. There were some randomization blocks at a given site that were not completely used; thus, the aggregated total number of subjects per treatment across surgery type and site were not the same. The inclusion/exclusion criteria may have resulted in a study population that is not completely representative of the general colorectal surgery population because subjects with certain conditions were not allowed to participate, such as those with repeat procedures or significant comorbidities. It should be noted that AEs defined a priori as potentially opioid related could also have been related to the surgical procedure or anesthesia received. Lastly, this study did not include an active comparator; it was designed to examine the safety and efficacy of meloxicam IV administered preoperatively, as at the time the study was initiated, only postoperative administration data were available. In a small Phase II study conducted in 50 patients undergoing laparoscopic abdominal surgery, pain relief following postoperative meloxicam IV $30 \mathrm{mg}$ once daily was similar to that provided by ketorolac IV administered every $6 \mathrm{~h}$ [30]. At the time of this writing, the relative effects of meloxicam IV and IV COX-2 selective agents have not been evaluated in the perioperative setting; however, the ability to administer meloxicam intravenously may be advantageous in these patients, who may be NPO or experiencing decreased gastrointestinal motility related to surgery.

\section{Conclusion}

The results of the current study indicate that incorporating meloxicam IV into an ERAS protocol, with administration beginning prior to surgery, generally is well tolerated, and associated with reductions in opioid use in patients who undergo colorectal surgery. These preliminary results suggest that meloxicam IV should be evaluated further in colorectal surgery trials that are adequately powered to assess between-treatment differences.

Supplementary data

To view the supplementary data that accompany this paper please visit the journal website at: www.futuremedicine.com/doi/suppl/10.2217/pmt-2020-0061

\section{Author contributions}

JD Silinsky and JE Marcet contributed to study design and implementation; managed subjects and helped with data acquisition; helped draft the article and/or critically reviewed it for important intellectual content; approved the final article. VR Anupindi, SU Karkare, DR Shah, W Du and A Freyer contributed to study design and implementation; provided guidance on data analysis and interpretation; helped draft the article and/or critically review it for important intellectual content; approved the final article. RJ Mack, SW McCallum and LK Black contributed to study design, data analyses plan and reporting of the data/results; provided guidance on data interpretation; helped draft the article and/or critically review it for important intellectual content; approved the final article.

\section{Acknowledgments}

The authors would like to thank CP Delaney, who participated in the design of the study and its implementation.

\section{Meeting presentations}

Limited portions of the material reported in this manuscript were accepted for presentation at the American Society of Colon and Rectal Surgeons (ASCRS) Annual Scientific Meeting in Boston, MA, 6-10 June 2020, and at Digestive Disease Week (DDW) 2020 in Chicago, IL, 2-5 May 2020. The ASCRS poster focuses on clinical outcomes and the DDW poster focuses on economic outcomes. 
and stockholders of Baudax Bio, formerly Recro Pharma; W Du is a consultant (statistical analysis) for Baudax Bio, formerly Recro Pharma. The authors have no other relevant affiliations or financial involvement with any organization or entity with a financial interest in or financial conflict with the subject matter or materials discussed in the manuscript apart from those disclosed.

Medical writing support was provided by B Fulton and S Martin of The Medicine Group, New Hope, PA, USA, and was funded by Baudax Bio Inc., formerly Recro Pharma, Malvern, Pennsylvania.

\section{Ethical conduct of research}

The authors state that they have obtained appropriate institutional review board approval or have followed the principles outlined in the Declaration of Helsinki for all human or animal experimental investigations. In addition, for investigations involving human subjects, informed consent has been obtained from the participants involved.

\section{Data sharing statement}

The authors certify that this manuscript reports original clinical trial data, NCT03323385. Individual, de-identified participant data will not be available. Data will be available to the public at https://clinicaltrials.gov/ct2/show/NCT03323385 for the duration of 2020.

\section{Open access}

This work is licensed under the Attribution-NonCommercial-NoDerivatives 4.0 Unported License. To view a copy of this license, visit http://creativecommons.org/licenses/by-nc-nd/4.0/

\section{References}

Papers of special note have been highlighted as: $\bullet$ of interest; $\bullet \bullet$ of considerable interest

1. Chemali ME, Eslick GD. A meta-analysis: postoperative pain management in colorectal surgical patients and the effects on length of stay in an Enhanced Recovery After Surgery (ERAS) setting. Clin. J. Pain 33(1), 87-92 (2017).

2. Lee L, Li C, Landry T et al. A systematic review of economic evaluations of enhanced recovery pathways for colorectal surgery. Ann. Surg. 259(4), 670-676 (2014).

3. Joshi GP, Schug SA, Kehlet H. Procedure-specific pain management and outcome strategies. Best Pract. Res. Clin. Anaesthesiol. 28(2), 191-201 (2014).

4. Li D, Jensen CC. Patient satisfaction and quality of life with enhanced recovery protocols. Clin. Colon Rectal Surg. 32(2), 138-144 (2019).

5. Basse L, Hjort Jakobsen D, Billesbolle P, Werner M, Kehlet H. A clinical pathway to accelerate recovery after colonic resection. Ann. Surg. 232(1), 51-57 (2000).

6. Delaney CP, Fazio VW, Senagore AJ, Robinson B, Halverson AL, Remzi FH. 'Fast track' postoperative management protocol for patients with high co-morbidity undergoing complex abdominal and pelvic colorectal surgery. Br. J. Surg. 88(11), 1533-1538 (2001).

7. Greer NL, Gunnar WP, Dahm P et al. Enhanced recovery protocols for adults undergoing colorectal surgery: a systematic review and meta-analysis. Dis. Colon Rectum 61(9), 1108-1118 (2018).

- This paper summarizes the benefits and limitations of enhanced recovery after surgery protocols for adults undergoing colorectal surgery using data from 25 trials.

8. Delaney CP. Chasing surgical value. Am. J. Surg. 213(3), 439-442 (2017).

9. Simpson JC, Bao X, Agarwala A. Pain management in enhanced recovery after surgery (ERAS) protocols. Clin. Colon Rectal Surg. 32(2), 121-128 (2019).

10. Nimmo SM, Foo ITH, Paterson HM. Enhanced recovery after surgery: pain management. J. Surg. Oncol. 116(5), 583-591 (2017).

11. Carmichael JC, Keller DS, Baldini G et al. Clinical practice guidelines for enhanced recovery after colon and rectal surgery from the American Society of Colon and Rectal Surgeons and Society of American Gastrointestinal and Endoscopic Surgeons. Dis. Colon Rectum 60(8), 761-784 (2017).

- These evidence-based clinical practice guidelines define "current best quality care for enhanced recovery after colon and rectal surgery".

12. Nafziger AN, Barkin RL. Opioid therapy in acute and chronic pain. J. Clinical Pharmacol. 58(9), 1111-1122 (2018).

13. Gupta A, Bah M. NSAIDs in the treatment of postoperative pain. Curr. Pain Headache Rep. 20(11), 62 (2016).

14. Chapman SJ, Garner JJ, Drake TM, Aldaffaa M, Jayne DG. Systematic review and meta-analysis of nonsteroidal anti-inflammatory drugs to improve GI recovery after colorectal surgery. Dis. Colon Rectum 62(2), 248-256 (2019).

15. Del Tacca M, Colucci R, Fornai M, Blandizzi C. Efficacy and tolerability of meloxicam, a COX-2 preferential nonsteroidal anti-inflammatory drug. Clin. Drug Investig. 22(12), 799-818 (2002).

- This older review summarizes the efficacy and tolerability of meloxicam. 
16. ANJESO [package insert]. Baudax Bio, Inc, Malvern, PA (2020).

17. Christensen SE, Cooper SA, Mack RJ, McCallum SW, Du W, Freyer A. A randomized double-blind controlled trial of intravenous meloxicam in the treatment of pain following dental impaction surgery. J. Clin. Pharmacol. 58(5), 593-605 (2018).

18. Pollak R, Gottlieb IJ, Hakakian F et al. Efficacy and safety of intravenous meloxicam in patients with moderate-to-severe pain following bunionectomy: a randomized, double-blind, placebo-controlled trial. Clin. J. Pain 34(10), 918-926 (2018).

19. Bergese S, Melson T, Candiotti $\mathrm{K}$ et al. A Phase III, randomized, placebo-controlled evaluation of the safety of intravenous meloxicam following major surgery. Clin. Pharmacol. Drug Dev. 8(8), 1062-1072 (2019).

20. Rechberger T, Mack RJ, McCallum SW, Du W, Freyer A. Analgesic efficacy and safety of intravenous meloxicam in subjects with moderate-to-severe pain after open abdominal hysterectomy: a Phase II randomized clinical trial. Anesth. Analg. 128(6), 1309-1318 (2018).

21. Singla N, Bindewald M, Singla $S$ et al. Efficacy and safety of intravenous meloxicam in subjects with moderate-to-severe pain following abdominoplasty. Plast. Reconstr. Surg. Glob. Open 6(6), e1846 (2018).

22. Palliative Meds Info. Opioid Conversion Chart. https://olh.ie/wp-content/uploads/2014/09/Opioid-Conversion-Chart.pdf

23. Joshi GP, Bonnet F, Kehlet H. Evidence-based postoperative pain management after laparoscopic colorectal surgery. Colorectal Dis. 15(2), 146-155 (2013).

24. Lovich-Sapola J, Smith CE, Brandt CP. Postoperative pain control. Surg. Clin. North Am. 95(2), 301-318 (2015).

25. Benyamin R, Trescot AM, Datta S et al. Opioid complications and side effects. Pain Physician 11(Suppl. 2), S105-S120 (2008).

26. White PF. What are the advantages of non-opioid analgesic techniques in the management of acute and chronic pain? Expert Opin. Pharmacother. 18(4), 329-333 (2017).

27. Viscusi ER, Gan TJ, Bergese $S$ et al. Intravenous meloxicam for the treatment of moderate to severe acute pain: a pooled analysis of safety and opioid-reducing effects. Reg. Anesth. Pain Med. 44, 360-368 (2019).

-. This pooled analysis summarizes evidence of the safety and opioid-reducing effects of intravenous meloxicam from seven Phase II and three clinical trials.

28. Huang Y, Tang SR, Young CJ. Nonsteroidal anti-inflammatory drugs and anastomotic dehiscence after colorectal surgery: a meta-analysis. ANZ J. Surg. 88(10), 959-965 (2018).

29. Modasi A, Pace D, Godwin M, Smith C, Curtis B. NSAID administration post colorectal surgery increases anastomotic leak rate: systematic review/meta-analysis. Surg. Endosc. 33(3), 879-885 (2019).

30. Singla N, McCallum SW, Mack RJ, Freyer A, Hobson S, Du W. Safety and efficacy of an intravenous nanocrystal formulation of meloxicam in the management of moderate to severe pain following laparoscopicabdominal surgery. J. Pain Res. 11, 1901-1903 (2018). 
